\title{
The Initial Results from the LITN of the FORMOSAT-3 TBB Program
}

\author{
Tung Yuan Hsiao ${ }^{1,2, *}$, Lung-Chih Tsai ${ }^{1,3}$, and Chao-Han Liu ${ }^{2}$ \\ ${ }^{1}$ Department of Information Technology, Hsing Wu College, Lin-Kou, Taiwan, ROC \\ ${ }^{2}$ Institute of Space Science, National Central University, Chung-Li, Taiwan, ROC \\ ${ }^{3}$ Center for Space and Remote Sensing Research, National Central University, Chung-Li Taiwan, ROC
}

Received 1 May 2007, accepted 3 December 2007

\begin{abstract}
Beginning in 2006 , four ground stations located at Chung-Li $\left(25.136^{\circ} \mathrm{N}, 121.539^{\circ} \mathrm{E}\right)$, TasoTun $\left(23.981^{\circ} \mathrm{N}, 120.697^{\circ} \mathrm{E}\right)$, CheCheng $\left(22.725^{\circ} \mathrm{N}, 120.544^{\circ} \mathrm{E}\right)$, and tropical Itu Aba Island $\left(10.06^{\circ} \mathrm{N}, 114.350^{\circ} \mathrm{E}\right)$ were set up to receive beacon signals from six FORMOSAT-3/COSMIC (Bernhardt et al. 2000) and other NNSS-like satellites. By applying differential Doppler technique on three quadrature components of the three-band beacon signals, we were able to obtain total electric contents (TEC) values and scintillation along the radio path through the ionosphere. Such measurements can be used to derive regional ionospheric irregularity and tomography. In this paper, we have confirmed the power patterns contributed from the transmitting antennas on board FS3/COSMIC and the ground-based receiving antennas. The multiplicative algebraic reconstruction technique (MART) algorithm has also been implemented to reconstruct ionospheric tomography images. Observations of the diurnal variations and the dynamics of equatorial anomaly showed that the equatorial anomaly appeared at $0800 \mathrm{LT}$, and then subsequently moved toward the Earth's poles. The north crest was strongest at $\sim 1300 \mathrm{LT}$ and its core was located at about $10^{\circ} \mathrm{N}$ of the magnetic latitude $\left(\sim 20^{\circ} \mathrm{N}\right.$ geographic latitude). Afterwards, the crest moved toward the equator after sunset and disappeared after midnight.
\end{abstract}

Key words: Total electron content (TEC), Equatorial anomaly, Ionospheric tomography, Beacon receiver

Citation: Hsiao, T. Y., L. C. Tsai, and C. H. Liu, 2009: The initial results from the LITN of the FORMOSAT-3 TBB program. Terr. Atmos. Ocean. Sci., 20, 261-271, doi: 10.3319/TAO.2007.12.03.01(F3C)

\section{INTRODUCTION}

The electron density distribution in the ionosphere exhibits a complicated structure and dynamic behavior that has been studied for many years. The ionosphere affects the reliability of satellite communications and the accuracy of radio astronomy. There are several techniques in measuring the ionospheric electron density from ground stations. The ionosonde, which is essentially a one-dimensional radar measures the maximum electron density in an upwardly direction. On the other hand, the incoherent scatter radar can measure ionospheric electron density in a three dimensional manner. However, as there are only eight or nine such radars in the world, limited coverage is achieved. Ionospheric tomography is another technique that can be used to obtain information about the ionospheric electron density. An ionospheric tomography system consists of a navigational

\footnotetext{
* Corresponding author

E-mail:TungYuanHsiao@gmail.com
}

satellite usually orbiting at a LEO altitude between 800 to $1200 \mathrm{~km}$, along with several ground stations positioned in a line directly under the LEO satellite's orbital path. A typical configuration for an ionospheric tomography system is shown in Fig. 1. The data measured at the ground stations are total electron content (TEC) values. The TEC data can be expressed as Eq. (1):

$T E C=\int_{L} N_{e} d s$

where the $N_{e}$ is the electron density and $L$ is the path between the receiver and the satellite. Thus, the electron densities could be inverted from TEC measurements as computing tomography (Austen et al. 1988). Ionospheric tomography systems are much less expensive than incoherent scatter radar systems and offer wider coverage. The practical feasibility of ionospheric tomography has been de- 


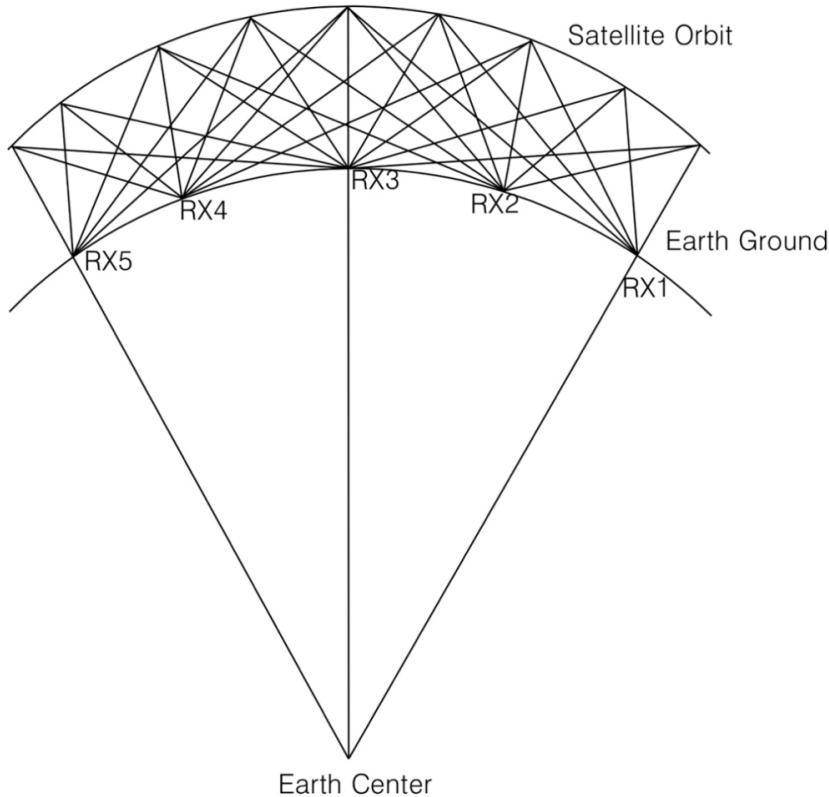

Fig. 1. Geometry of an ionospheric tomography system.

monstrated in several studies (Na and Lee 1991; Fremouw et al. 1992; Kunitsyn and Tereschenko 1992; Pryse and Kersley 1992; Kersley and Pryse 1994; Kronschnabl et al. 1995; Pakula et al. 1995).

As shown in Fig. 1 all paths are linked between the two concentric circles formed by the surface of the Earth and the satellite path; none of the paths lie across the ionosphere. This is a fundamental limitation associated with ionospheric tomography systems having poor vertical resolution. However, the poor vertical resolution can be improved through a careful design of the tomography reconstruction algorithm. Many techniques have been used for ionospheric tomography reconstruction. In other words, most tomographic problems are ultimately solved via systems of linear equations (SLE). Hence, it is important to choose a proper algorithm in solving the SLE. Up until now, the most commonly used algorithms that are based upon pixels include - algebraic reconstruction technique (ART), simultaneous iterative reconstruction technique (SIRT), and multiplicative algebraic reconstruction technique (MART). ART, SIRT, and MART need a priori information in initially guessing the solution. MART has an advantage over ART in determining the electron densities, as it avoids unreasonable negative values, and it has been used in this study. Raymund et al. (1994) and Tsai et al. (2002) proposed an improved MART algorithm that used a priori information contained in a set of model or simulated ionospheres, such as the IRI (International Reference Ionosphere) model, in supplying the information contained in the data. Fehmers (1994), on the other hand, proposed another algorithm that employed smooth constraints instead of model ionospheres. A technique called phase-difference tomography was also introduced in re- moving calibration offsets associated with the receivers (Kunitsyn and Tereschenko 1992; Kunitsyn et al. 1994). In this paper, we have applied the IRI model 2001 in obtaining a rational initial guess and implemented the MART algorithm on the TEC data measured by the new Low-latitude Ionosphere Tomography Network (LITN) chain in reconstructing ionospheric tomography images. We have to firstly verify the recorded beacon signal magnitudes contributed from the power patterns of the ground receiving antenna and the transmitting antenna on board FS3/COSMIC. In fact, the tri-band transmitting antenna on board $\mathrm{FS} 3 / \mathrm{COSMIC}$ has a similar design as the receiving antenna of LITN ground stations. Secondly, from the tomography results of the new LITN, we observed the diurnal variations and the dynamics of the equatorial anomaly, where the north crest appeared at $\sim 0800$ LT and then moved toward the Earth's poles. The north crest was strongest at $\sim 1300$ LT and its core was located at about $10^{\circ} \mathrm{N}$ of the magnetic latitude $\left(\sim 20^{\circ} \mathrm{N}\right.$ geographic latitude). It subsequently moved toward the equator after sunset and disappeared after midnight.

\section{INSTRUMENTS AND OBSERVATION METHODS}

\subsection{Ionospheric Tomography System (ITS) Overview}

There are currently many ionospheric tomography networks located in the world, where they are mostly focused on the polar and high latitude regions in Europe and midlatitude in North America. Australia's ionospheric tomography network is the only system located in Earth's southern region and within the mid-latitudes. Meanwhile, only the Indian and LITN networks cross the magnetic equators, which concentrate on equatorial anomaly dynamics. The old LITN had operated from 1994 to 1997. It was later shut down, as the US government turned off the radio beacon signals. Thereafter, in order to receive the beacon radio signals from the six FS3/COSMIC satellites, which are named: FM1, FM2, FM3, FM4, FM5, and FM6 individually and were successfully launched on 15 April in 2006, we established three ITS (Ionospheric Tomography System) stations at Chung-Li, TasoTun, and CheCheng in 2006 too. The ITS systems were built by NWRA (Northwest Research Associated, Inc.) and can receive beacon signals not only from FS3/COSMIC satellites but also NNSS-like (Navy Navigation Satellite System) satellites including OSCAR series, GFO, RADCAL, and COSMOS series. In December 2006, a fourth station was set up on Itu Aba Island $\left(10^{\circ} 23^{\prime} \mathrm{N}\right.$, $\left.114^{\circ} 2^{\prime} \mathrm{E}\right)$. We need to further extend the station chain either southward or northward, as the network is deemed slightly inadequate for the tomography reconstruction. Before 2008, we plan to set up nine stations equipped with the ITS30 coherent beacon receivers along longitude $120^{\circ} \mathrm{E}$ and latitude from $40^{\circ} \mathrm{N}$ to $6^{\circ} \mathrm{S}$. This will include Beijing, Shanghai, Chung-Li, TasoTun, CheCheng, ltu Aba Island, Manila, Pare-Pare, and Djakarta, as shown in Fig. 2. By then, the 


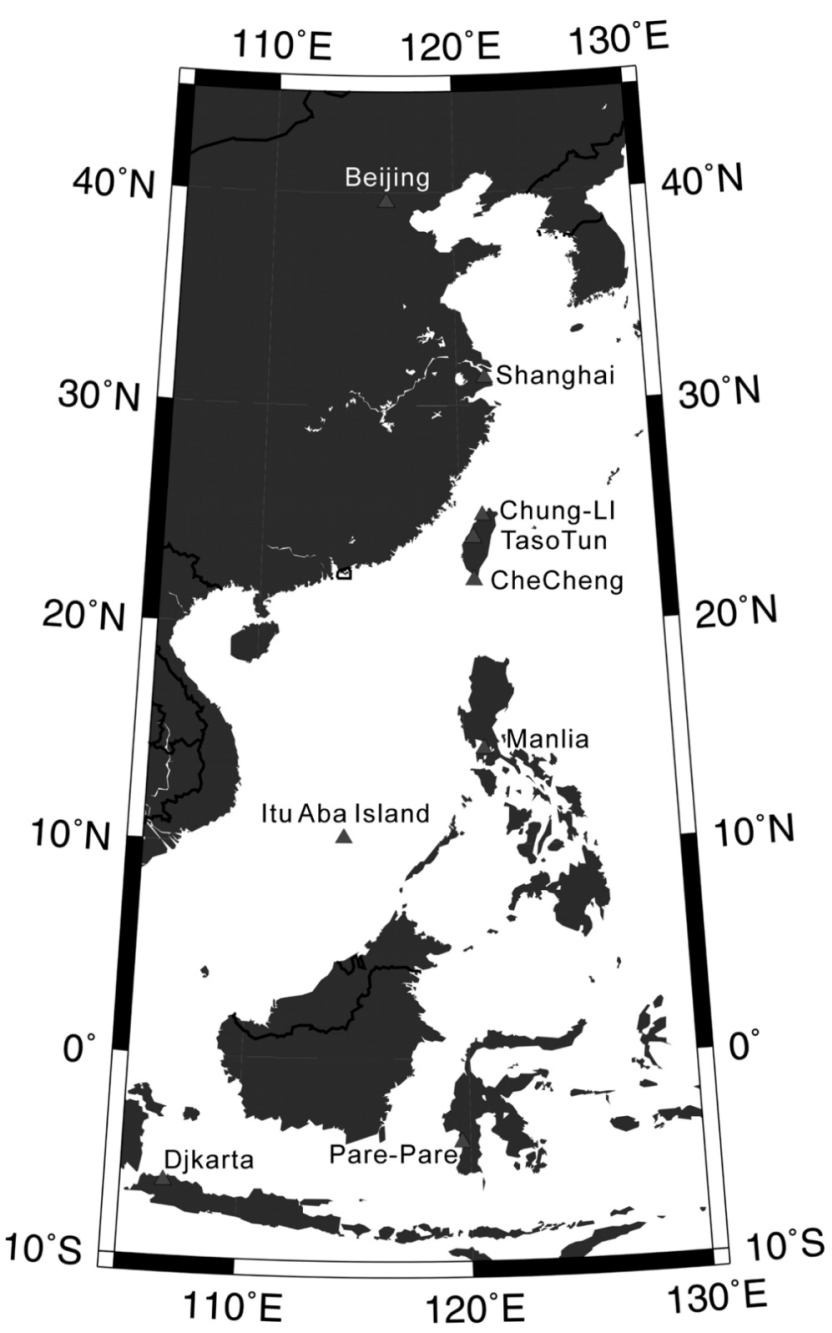

Fig. 2. The Beijing, Shanghai, Chung-Li, TaoTung, CheCheng, ltu Aba Island, Manila, Pare-Pare, and Djakarta ground stations in the new LITN.

scanning region will encompass the northern mid-latitude and the equator areas.

The three-band radio signals are transmitted from the TBB transmitter on board FS3/COSMIC, travel through the ionosphere and atmosphere, and are received by the groundbased ITS30 stations, independently. The three bands of FS3/COSMIC beacon signal are 150, 400, and 1066.7 MHz. Figures 3 and 4 show the simplified block diagram of the ITS30 receiver. The ground-based receivers are essentially composed of two parts including mixer module and Detector/PLL module and have various components, which include $1^{\text {st }}$ down converter (or $1^{\text {st }}$ IF band-pass filters), $1^{\text {st }}$ local oscillator, $2^{\text {nd }}$ down converter, $2^{\text {nd }}$ local oscillator, $2^{\text {nd }} I F$ filter, and logarithmic amplifier (or phase PLL demodulator). The principle of the superheterodyne is the use of the $50 \mathrm{KHz}$ as the base band. The sampling rate of the ADC converter is $50 \mathrm{~Hz}$, i.e., the low pass filter is $25 \mathrm{~Hz}$. The central control computer can modify the mixer module's gain step (one step is one $\mathrm{dB}$ ) and the controlling voltage for tuning the Doppler frequency, when the beacon frequencies are known and whenever the radio frequency changes in the wake of a satellite's moving speed.

\subsection{The Differential Doppler Technique and Relative Slant TECs}

The differential Doppler technique (Gringauz 1958) examines the effect of the ionosphere on the phase of two sinusoidal signals of frequencies $f_{1}$ and $f_{2}$. By substituting the phase paths for two phases, the path difference $\Delta \varphi$ can be written as:

$\Delta \varphi=\frac{40.3 f_{1}}{c}\left(\frac{1}{f_{2}^{2}}-\frac{1}{f_{1}^{2}}\right) \int N_{e} d s$

The difference of the phase paths is therefore related to the electron density, due to the total electron content (TEC) along the propagation path. Without the effect of the ionosphere, this weighted difference would be zero. In light of the various ambiguities that are introduced by the phase offset in the receivers, the differential phase should generally be rewritten to account for the unknown phase offset $\varphi_{0}$ as:

$\Delta \varphi=k \int N_{e} d s \pm \varphi_{0}$

Using a large number of line integral measurements, such as phase data obtained via Faraday rotation or Differential Doppler, slant TEC (STEC) values can be determined by phase path differences, and because of an unknown phase offset the STECs are relative.

\section{COMPARISONS OF SIMULATED ANTENNA PATTERNS AND ACTUAL SIGNAL MAGNITUDES}

It is well known that the Numerical Electromagnetic Code (NEC) program (Lawrence Livermore Laboratory 1977) can be used to simulate the electromagnetic response of general structures. The Numerical Electromagnetic Code, which was written by Gerald Burke, is a popular antenna modeling code for wire and surface antennas and scatterers for antenna designers - amateur and ham radio users. Models can include wires buried in a homogeneous ground, insulated wires and impedance loads. The code is based on the method of the moment's solution of the electric field integral equation for thin wires, and the magnetic field integral equation for closed conducting surfaces. Users can utilize the model more easily and rapidly, due to the Third Party Software Company or the friendly interface of the NEC model designed by amateur radio users. In this paper, we use this model to simulate the power 


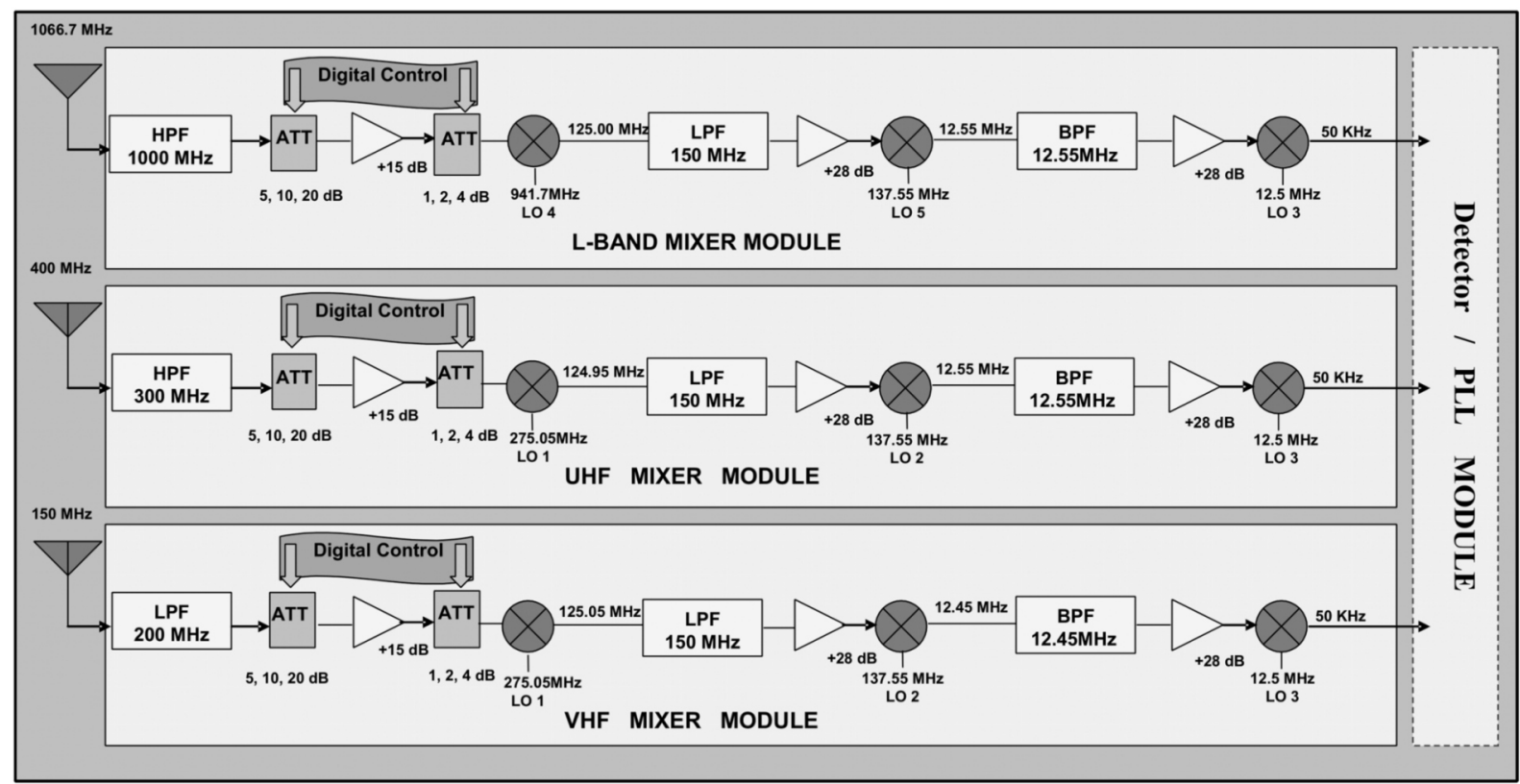

Fig. 3. The mixer module. There are three down converters in shifting the three band RF radio signals to the base band (50 KHz).

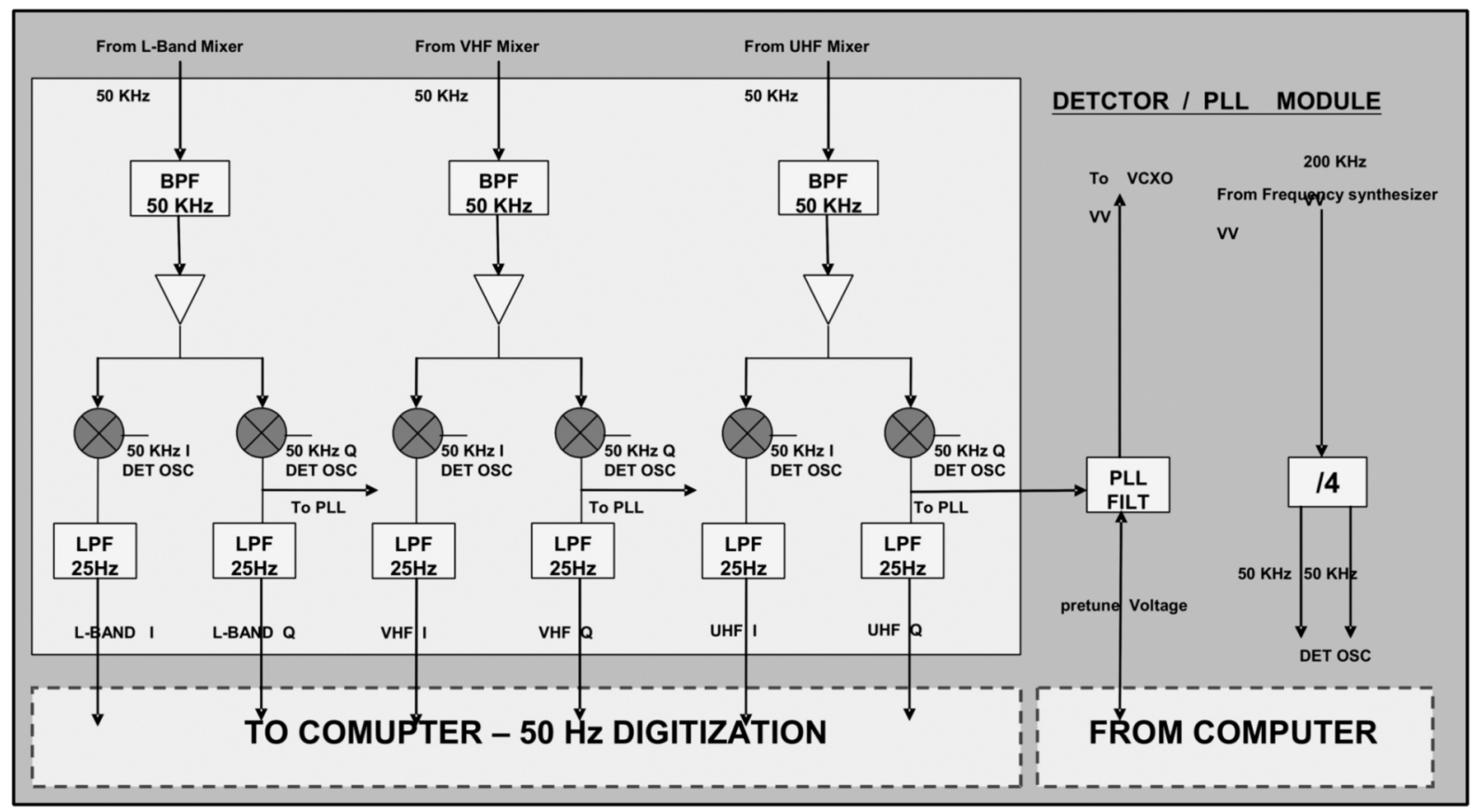

Fig. 4. The Detector module. The base band signals transforms into the in-phase (I) and quadrature (Q) channels. $50 \mathrm{~Hz}$ data of six channels are used for the VHF, UHF, and L-Band IQ channels.

patterns of the ITS30 ground-based receiving antenna and of the TBB transmitting antennas on board FS3/COSMIC.

In order to check the ITS30 receiving antenna status, we simulated the ITS30 receiving system by the NEC model. The design of the cross dipole for the ITS30 antenna Fig. 5a has a symmetrical circuit pattern in the horizontal plane, which is presented in Fig. 5b. This is important for the ITS30 stations, as the satellites will not appear at the fixed tracks and azimuth angles. The antenna gain at the horizontal plane is about 3.48 to $3.99 \mathrm{dBi}$ for 150,400 , and $1066.7 \mathrm{MHz}$. As for the vertical plane, they are depicted in Figs. $6 a, b$, and c for the three bands. The $\mathrm{dBi}$ is the gain in decibel referenced to an isotropic radiator. An isotropic radiator is a theoretical antenna with equal gain to all points on isotropic sphere. 
(a)

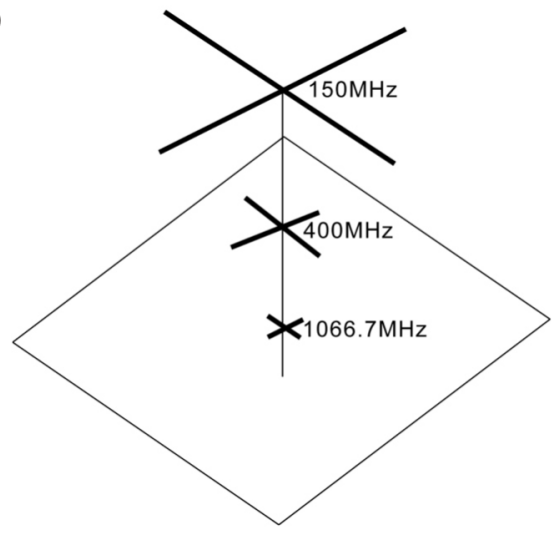

(b)

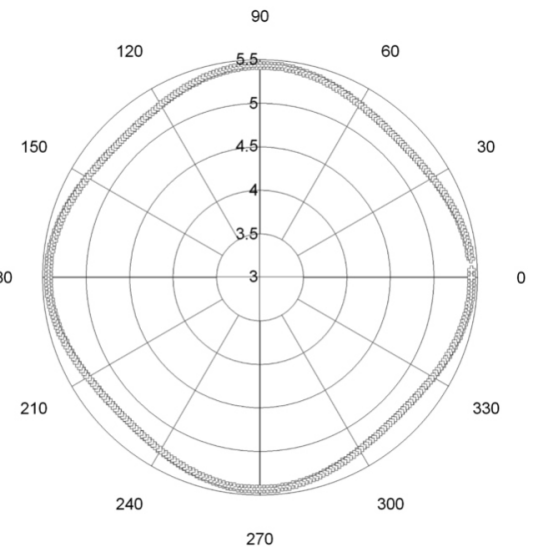

Fig. 5. (a) The ITS30 antenna design. (b) The simulation of the ITS30 antenna in the horizontal plane.

(a)

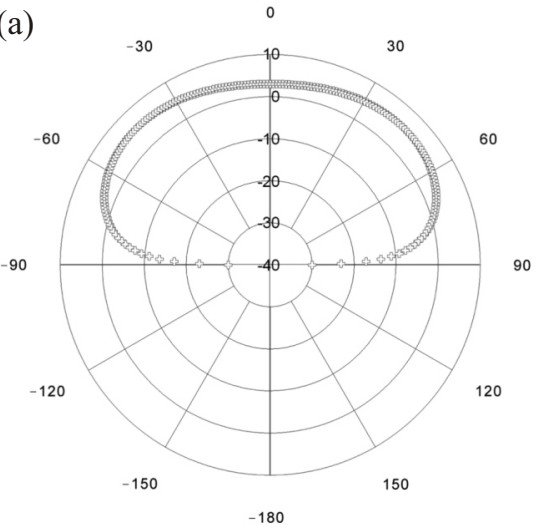

(b)

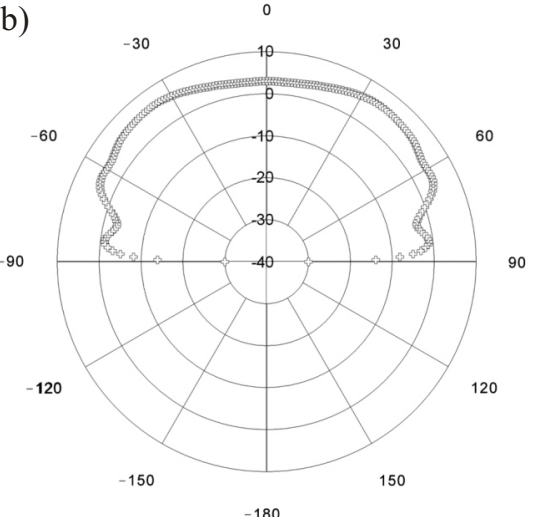

(c)

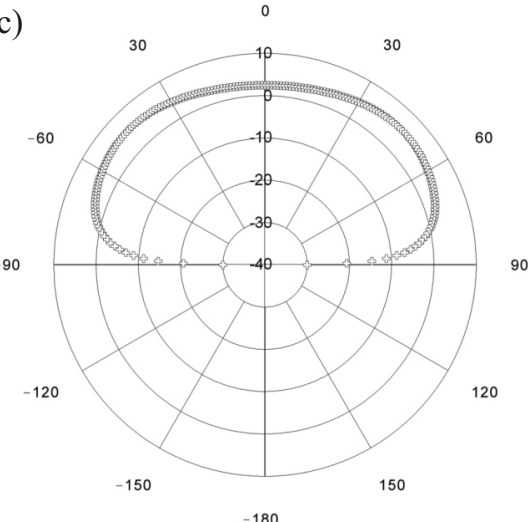

Fig. 6. The simulation of the ITS30 antenna in the vertical plane. (a) $150 \mathrm{MHz}$ (b) $400 \mathrm{MHz}$ (c) $1066.7 \mathrm{MHz}$.

The antenna gain is roughly $0 \mathrm{dBi}$ when the zenith angle is greater than 15 degrees or smaller than -15 degrees. Moreover, the maximum antenna gain at 45 degrees is about $5 \mathrm{dBi}$. It means the receiver will get $5 \mathrm{~dB}$ gains at 45 degrees; zero degree more than at the \pm 15 degrees. It is slightly incorrect at 1066.7 MHz, due to the suppression of the antenna elements at 150 and $400 \mathrm{MHz}$. Therefore, the simulation of the antenna pattern for $1066.7 \mathrm{MHz}$ is a little varied. In practice, the ITS30 receivers usually start to lock the signals at about 5 degrees if there is no blocking in the path of the signals near the vicinity of the ITS30 antenna. Therefore, the crossed dipole antenna is very suitable for the ITS30 receiver.

A common use of the quantization is converting a discrete signal (a sampled continuous signal) into a binary form. Both of these steps (sampling and quantizing) are performed in ADC converters with the quantization level specified in bits. The quantization level for the ITS30 was 12 bits, resulting in the dynamic range of the ITS30 being $36 \mathrm{~dB}$. Therefore, it is feasible to utilize the receiving data of the ITS30 in measuring the transmitting antenna power gain from satellites when the elevation angle is 90 degrees. A 90-degree elevation angle is necessary to describe the trans- mitting antenna power gain for receiving ground stations, in order to see the entire power distributions from the satellite in the vertical plane. Unfortunately, data that contain 90degree elevation angles need to be picked up by FS3/COSMIC satellites, as their tracks are not usually directly above the ground stations. Figure 7 is a typical example of such a

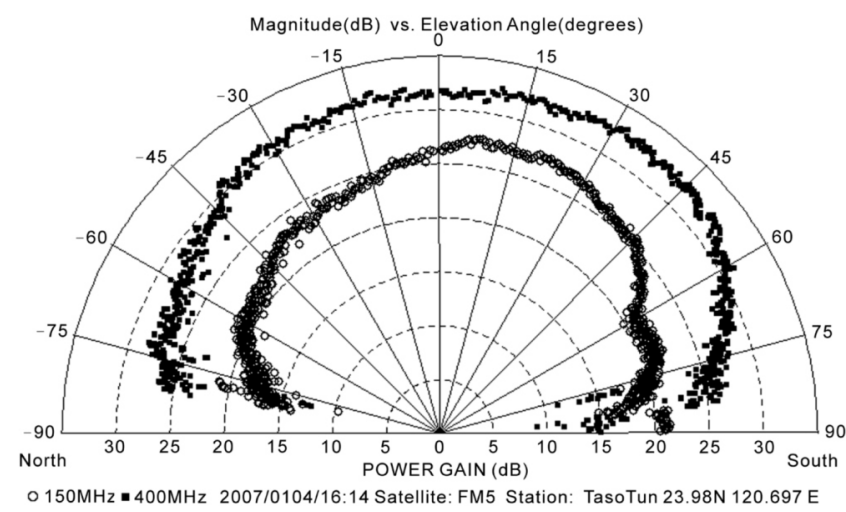

Fig. 7. The receiving data in the vertical plane of FORMOSAT 3's No. 5 satellite (FM 5) at 16:14 on 4 June 2007 for TasoTun station. 
situation. It shows the result of FS3/COSMIC's No. 5 (FM 5) satellite at 16:14 on 4 June 2007 for the TasoTun station. Based on simulations of the receiving antenna, we know that the zenith angles falling between \pm 75 degrees are reliable. The data is slightly spread out when the zenith angles are not between \pm 75 degrees, even as the ITS30 locks the signals from the satellites. The circular pot in Fig. 7 illustrates the $150 \mathrm{MHz}$ power pattern, and the squared pot represents the
$400 \mathrm{MHz}$ power pattern. We compared actual transmitting antenna measurements (Bernhardt and Siefring 2006) with data from the NRL (Naval Research Laboratory), which is the transmitting antenna designer of FS3/COSMIC, as shown in Figs. 8a and b. Moreover, the beacon transmitting antenna pattern of $150 \mathrm{MHz}$ presented in Figs. 8a and b shows the antenna at $400 \mathrm{MHz}$. Comparison of the power (dB) and azimuth angle for FM5 are revealed in Fig. 8. The
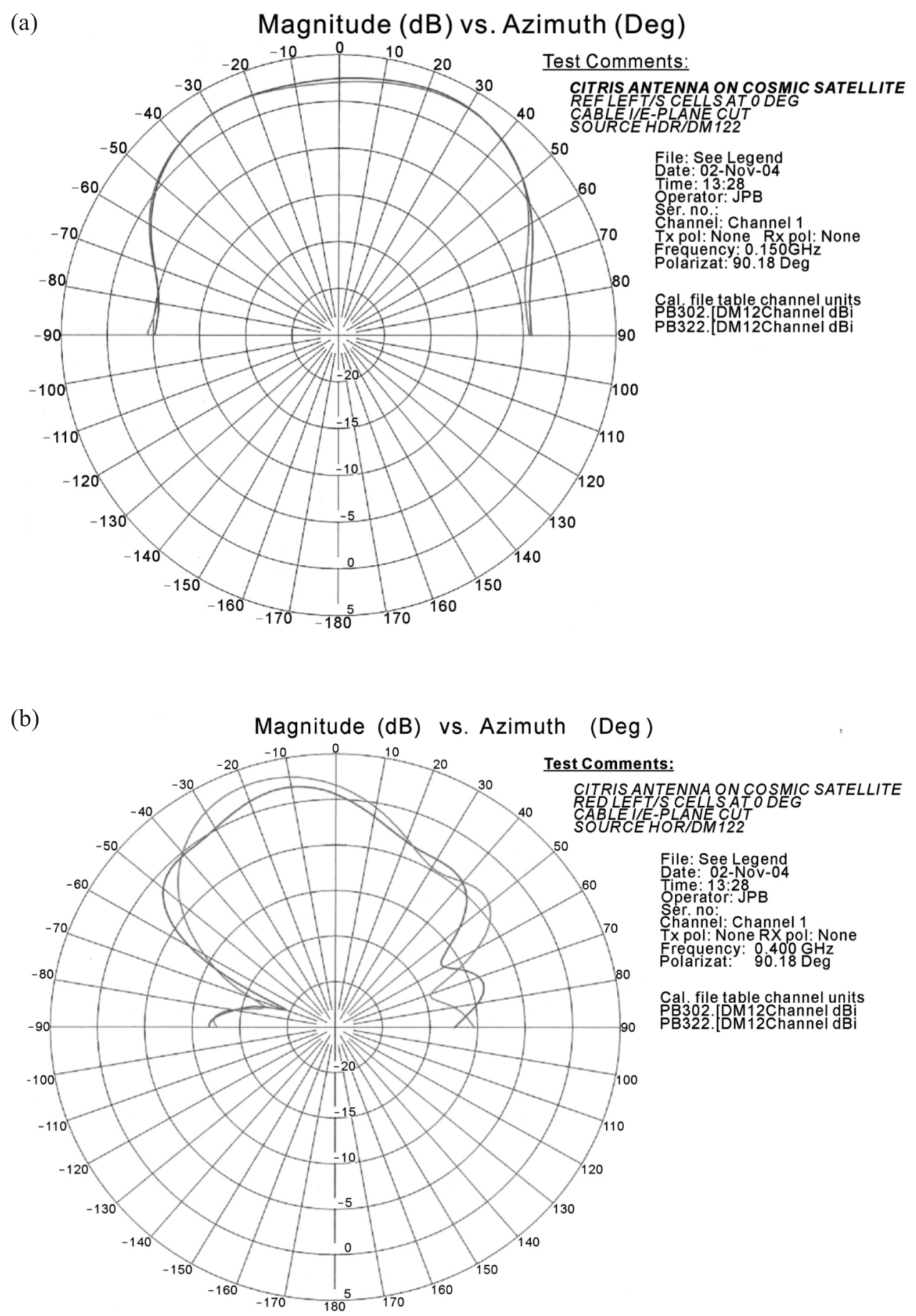

Fig. 8. Actual antenna measurements from NRL (Naval Research Laboratory). The two lines present the two measurements at different times. (a) $150 \mathrm{MHz}$ and (b) $400 \mathrm{MHz}$. 
azimuth angle in Fig. 8 depicts the different positions of our receiving data in the zenith angle in account of the axis measurement, which is contrary to the NRL figures. Nevertheless, they represent the same meaning. The antenna pattern is fairly similar to $150 \mathrm{MHz}$ in Figs. 6a and 7. Furthermore, the receiving data in Fig. 7 has a 60-degree gap to the right side, revealing a shape that is also similar to the actual measurements in Fig. 8b. Therefore, the beacon transmitting antenna on FORMOSAT 3 is seen to be working properly now. Moreover, the ITS30 antenna system is sensitive enough in carrying out the tri-beacon mission of FS3/COSMIC. A difficulty exists for comparison of the antenna pattern at 1066.7 MHz since this signal is only available on special request because the 150.012 and $1066.752 \mathrm{MHz}$ mix inside the GPS receiver on FS3/COSMIC to produce interference.

\section{THE MART ALGORITHM AND RESULTING TOMOGRAPHY}

A set of STEC measurements on the line-of-sight paths linked from a LEO satellite to ground-based receivers can form the following matrix as Eq. (4):

$Y=A x$

where $Y$ is a column of m measurements for STEC, $x$ is a column of n electron density unknowns for cells in the targeted ionosphere region, and $A$ is an $m \times n$ normal matrix with $a_{i l}$. Basically, the MART algorithm is iterated cyclically and can be implemented as the following Eq. (5):

$$
x_{l}^{k+1}=x_{l}^{k} \cdot\left(\frac{y_{i}}{\sum_{l=1}^{n} a_{i l} \times x_{l}^{k}}\right)^{\lambda_{k} \times a_{i l} / \sqrt{\sum_{l=1}^{n} a_{i l} \times a_{i l}}}, l=1, \ldots \ldots, n
$$

where $y_{i}$ is the $i$ th observed STEC in a column of m measurements, $x_{l}$ is the $l$ th resulted cell electron density in a column of n unknowns, $a_{i l}$ is the length of link $i$ that lies in cell $l, \lambda_{k}$ is the relaxation parameter at the $k$ th iteration with $0<\lambda_{k}<1$, and the inner product of the vectors $x$ and $a_{i}$ is thus the simulated STEC for the $i$ th path. The electron density matrix $x$ is corrected through an iteration by a ratio of the measured STEC and the simulated STEC with a relaxation parameter of $\lambda_{k}$. It is noted that any iterative algorithm requires an initial condition before the iteration begins. Consequently, the MART algorithm needs a priori information in the form of an initial guess for the solution too. With minimal ground receivers over a limited range of zenith angles for data collection, the measured STEC data may not be sufficient for the tomography inverse problem. Due to poor geometry, the initialization is extremely im- portant for the tomographic reconstruction. In practice, the closer the initial condition to the true electron density distribution is, the more accurate the reconstruction will be. In this study, the IRI-2001 model is served as an initial guess for the tomographic reconstruction in studying the MART algorithm.

In this study, we applied the data from four ITS30 ground receiver stations at Chung-Li, TasoTun, CheCheng, and ltu Aba Island for ionospheric tomography. The location of the new LITN is within the equatorial and low-latitude region. We have successfully presented the initial results from the new LITN on 26 to 31 December of 2006, and confirmed the earlier research results with the old LITN. A typical example of daytime reconstruction image is shown in Fig. 9a. The scanning areas of signals are indicated in the covered areas by the dashed-line in Fig. 9a. There are two main areas at $22 \sim 25^{\circ} \mathrm{N}$ latitudes and near $10^{\circ} \mathrm{N}$ latitude. And we can see that electron accumulation causes equato-

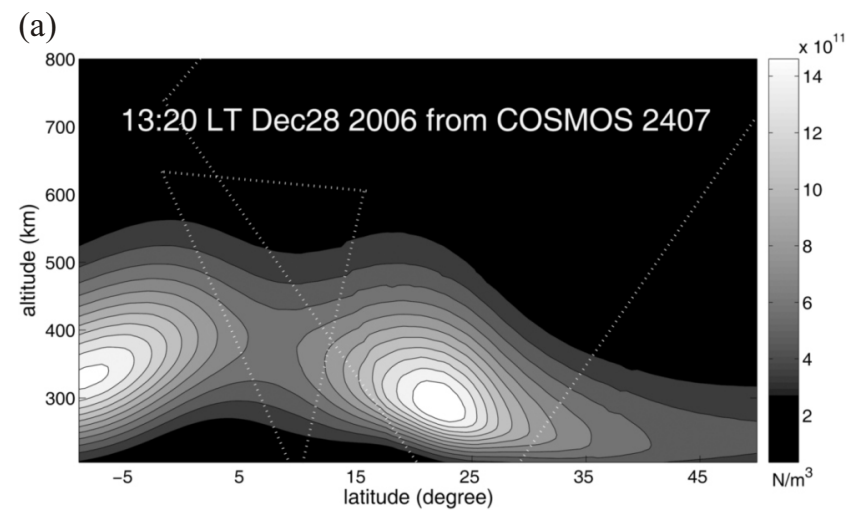

(b)

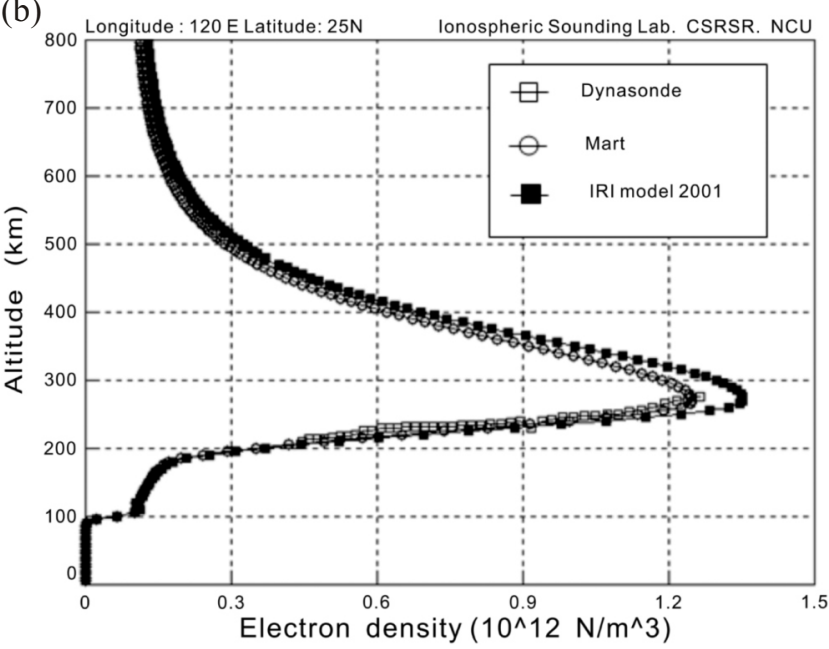

Fig. 9. (a) The ionospheric tomography result by MART at 1320 LT 28 December 2006. The areas in the dash-line are the scanning areas from the new LITN ITS30 stations. (b) The comparisons of electron density profiles above Chung-Li $\left(121.1^{\circ} \mathrm{E}, 24.5^{\circ} \mathrm{N}\right)$. The square dots show the Ne profile of IRI-2001 model, the circular dots present the Ne profile after MART algorithm processing, and the hollow square dots show the result from the Chung-Li dynasonde. 
rial anomaly (EA) and the north crest occurred at a latitude of $\sim 20^{\circ} \mathrm{N}$. To verify the tomography results after MART algorithm, independent data from the Chung-Li dynasonde has been used and compared to the vertical electron density profiles obtained from the new LITN. Figure $9 \mathrm{~b}$ presents the vertical electron density profiles from IRI-2001 model (hollow square dots), MART algorithm (circular dots), and Chung-Li dynasonde (square dots). The circular dots and hollow square dots are much closer than hollow square dots, which are from the forecast of IRI-2001 model. Comparing to the dynasonde data, peak electron density after MART algorithm has $4 \%$ fractional error, and the corresponding peak density height has $3 \%$ fractional error.

Figure 10 present prominent features of daytime equatorial anomaly from a series of tomography images obtained on 28 and 29 December 2006 and inverted by the beacon signal measurements from RADCAL, COSMOS2407, FM1, FM5, OSCAR23, and OSCAR31 satellites individually. Crests of the equatorial anomaly obscurely appeared at $\sim 0830$ LT of 28 December 2006, as shown in Fig. 10a. Af- ter two hours, electrons were accumulated, and Fig. 10b shows clear crests at about $18^{\circ} \mathrm{N}$ (the north crest) and $5^{\circ} \mathrm{N}$ (the south crest). It is noticed that the south crest were stronger than the north crest, while the formation of the north core equatorial anomaly is faster than the south core equatorial anomaly. From early studies (Moffett 1979; Andreeva 2000; Yeh 2001; Franke 2003), the trans-equatorial neutral winds transport ionization along the magnetic field line from one hemisphere to the other causing asymmetries in both peak electron densities and peak altitudes in the equatorial anomaly. Unfortunately, we were unable to observe the south crest at about $10^{\circ} \mathrm{S}$ during the afternoon, as the most southern Itu Iba Island station could not scan those areas. The grid values were contributed by initial guesses from the IRI 2001 model. In order to obtain a definite result, we focused only on data that was valid for the north equatorial anomaly in this paper. As shown in Figs. 10c, g, and h, we see that the north anomaly crests were strongest between 1200 to 1400 LT of 28 and 29 December and were showing a characteristic tilt approximately aligned along the geomagnetic field lines. Further- (a)
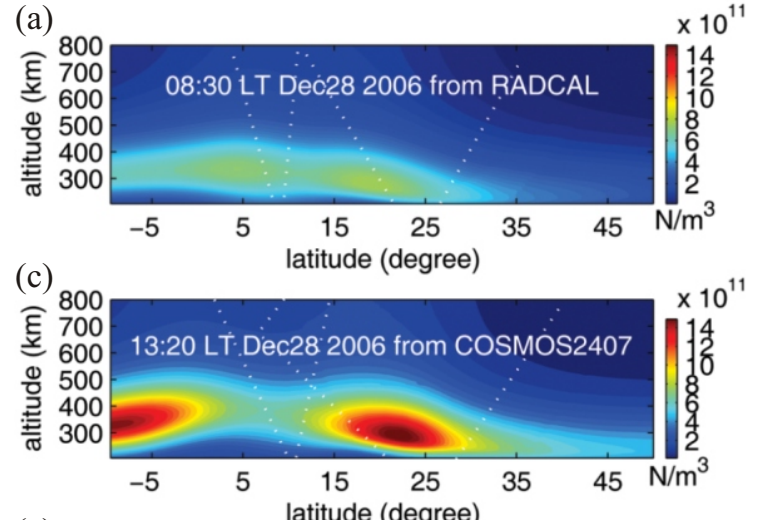

(e)

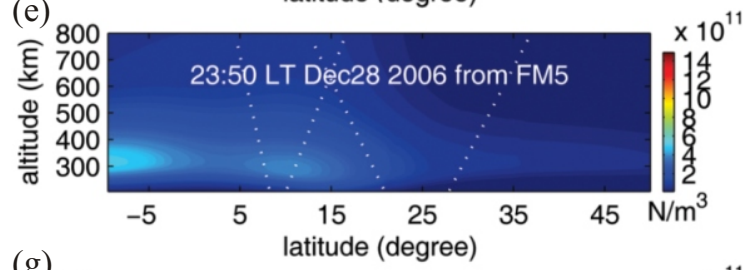

(g)
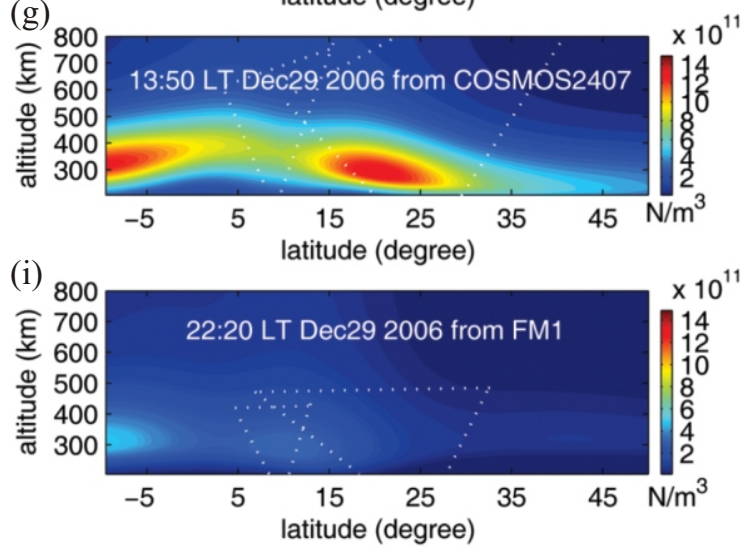

(b)

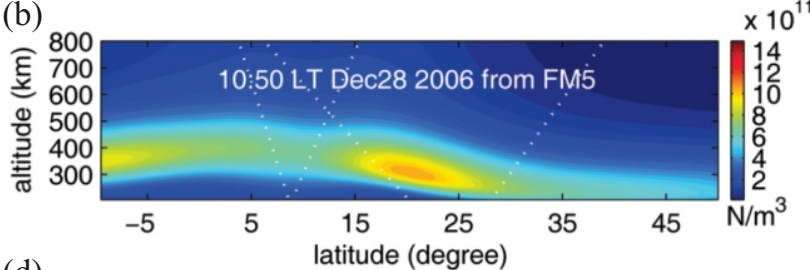

(d)
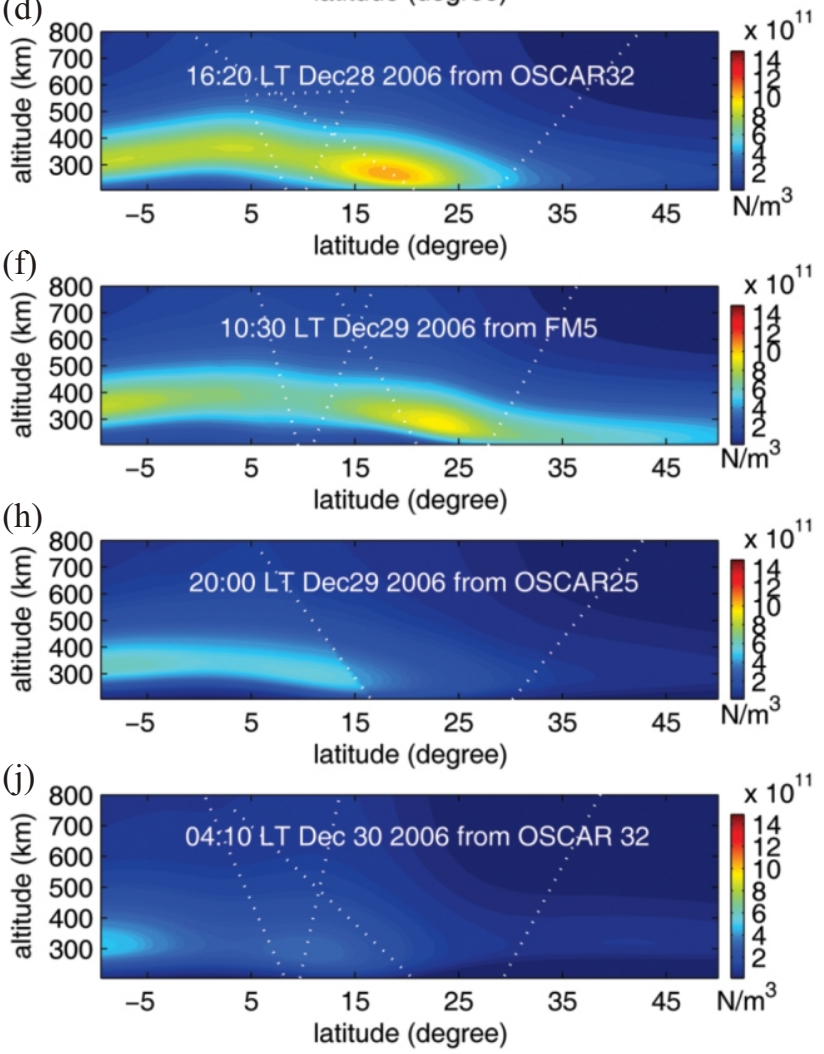

Fig. 10. The ionospheric tomography results obtained by the new LITN from 28 to 29 December in 2006. 
more, the north crest cores were located stably at $20^{\circ} \mathrm{N}$ within 1200 to 1400 LT. Thereafter, the north crests weakened and slowly receded equatorward. In Fig. 10d, we see the north crest core receding to $19^{\circ} \mathrm{N}$ at $1620 \mathrm{LT}$. Thereafter, as shown in Figs. 10e, i, and j, the north crests were very weak but still existed during 2000 to 2220 LT, and their cores were positioned approximately at $10^{\circ} \mathrm{N}$. The crests disappeared at about $0400 \mathrm{LT}$ as shown in Fig.10f.

Using such ionospheric tomographies, we can also analyze the motion of the equatorial anomaly observed during 26 to 31 December 2006 while $\mathrm{Kp}<3$. Figure 11 presents the diurnal variations of the electron density and corresponding latitude of the north crest cores. The equatorial anomaly happened at $\sim 18^{\circ} \mathrm{N}$ and $\sim 0800 \mathrm{LT}$. Thereafter, the north crest moved poleward by a speed of $\sim 1^{\circ}$ per hour. The strongest north crest happened at $20^{\circ} \mathrm{N}$ and at $1400 \mathrm{LT}$. And then started to recede equatorward with a speed of $\sim 1^{\circ}$ per hour, too. However, maximum electron density occurred at $1300 \mathrm{LT}$. The reason for this relation is that the crest reversed and weakened slowly equatorward after $1400 \mathrm{LT}$, but the fountain effects still were very strong. Hence, most electron accumulation occurred at 1300 LT instead. Meanwhile, the height of the north crest core began to lift after the crest happened at $\sim 0800$ LT as in Fig. 12. The core moved poleward and its height increased simultaneously and vice versa. The variances of them strongly hint that the fountain effects are supported mainly by the strength of $\vec{E} \times \vec{B}$ drift if the magnetic activity is low. While many sources of aeronomic and solar origin (Kelly et al. 2003) are good candidates for causing such variations, the electro-dynamic mechanism is especially relevant for the equatorial ionosphere. Previous

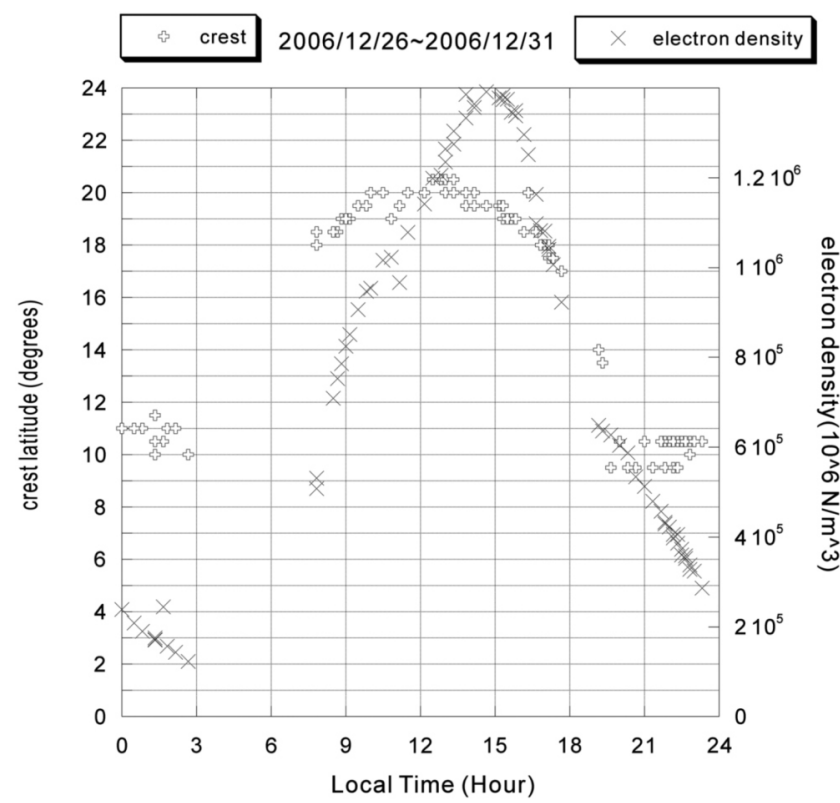

Fig. 11. The diurnal variations of electron density and latitude for the north crest core. studies have shown the importance of the dynamo field in controlling the behavior of the equatorial ionosphere. The daytime dynamo generated eastward electric field combined with the northward geomagnetic field lifting the equatorial ionosphere to $700 \mathrm{~km}$ and higher $(1000 \mathrm{~km})$. After losing momentum, the electrons become diffuse along the field lines to either side of the equator to form two crests (Hanson and Moffett 1966; Bailey et al. 1997).

\section{CONCLUSION AND FUTURE WORKS}

In this paper, we have simulated and verified the power patterns of the ITS30 receiving antennas and the TBB beacon transmitting antenna on board FS3/COSMIC. The TBB beacon transmitting antenna on FS3/COSMIC satellites worked well in supporting the ITS30 observations, including scintillations and ionospheric tomography (Bernhardt et al. 2000). We confirmed the initial results of the diurnal variations of the north crest with the old LITN data, as well as implemented the MART algorithm for ionospheric tomography. We observed the diurnal variations and the dynamics of the equatorial anomaly, which happened at $\sim 0800$ LT. Afterwards, it was seen progressing toward the Earth's poles. The strongest equatorial anomaly happened at $1300 \mathrm{LT}$ and its north crest core was located at about $20^{\circ} \mathrm{N}$ of geographic latitude $\left(\sim 10^{\circ} \mathrm{N}\right.$ of magnetic latitude). The north crest moved equatorward after $1400 \mathrm{LT}$ and subsequently disappeared after 0300 LT. The initial results from the new LITN proved to be satisfactory. The next step in our study will be finishing the setup of other ITS stations in the new LITN for more in-depth research in the dynamics behind

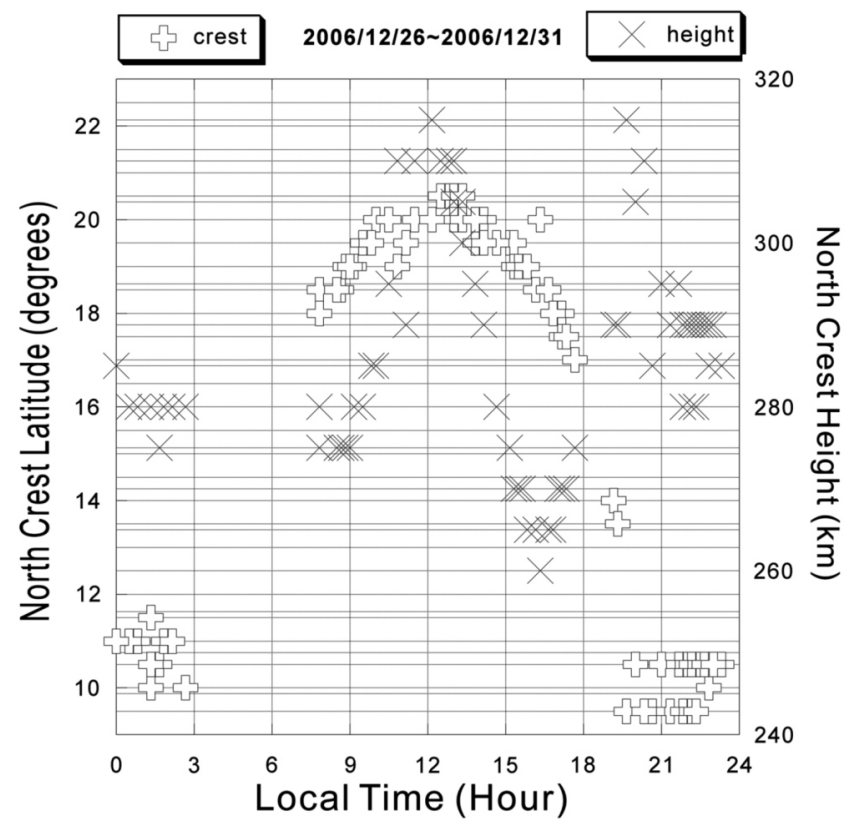

Fig. 12. The diurnal variations of height and latitude for the north crest core. 
equatorial anomalies. Meanwhile, we also plan to extend the distribution of the new LITN north to Beijing and south to Pare-Pare before 2008 and combine the TBB ground receiver data with the other two sensors on the COSMIC beacon, GOX and TIP (Bernhardt et al. 1998) in the future to obtain more complete observations.

Acknowledgments This work is supported by NSC942111-M-008-031-AP3 from the National Science Council of the Republic of China. We are also very grateful for comments made by those who reviewed the paper. Furthermore, we appreciate Francis Smith of NorthWest Research Associates, Inc, for his contribution relating to ITS30, which he designed. We also thank the Naval Research Laboratory for measured TBB antenna pattern data provided by the Plasma Physics Division of the Naval Research Laboratory in Washington, DC. Finally, we appreciate help in reconstruction of 30 stations from Jan-Jung Li, who is the director of the public service center at the National Museum of Marine Biology and Aquarium, and professor Chi-Ruei, Huang at Nan Kai Institute of Technology.

\section{REFERENCES}

Andreeva, E. S., S. J. Franke, K. C. Yeh , and V. E. Kunitsyn, 2000: Some features of the equatorial anomaly revealed by ionospheric tomography. Geophys. Res. Lett., 27, 24652468, doi: 10.1029/1999GL003725. [Link]

Austen, J. R., S. J. Franke, and C. H. Liu, 1988: Ionospheric imaging using computerized tomography. Radio Sci., 23, 299-307, doi: 10.1029/RS023i003p00299. [Link]

Bailey, G. J., N. Balan, and Y. Z. Su, 1997: The Sheffield University plasmasphere ionosphere model-A review. $J$. Atmos. Sol.-Terr. Phys., 59, 1541-1552, doi: 10.1016/ S1364-6826(96)00155-1. [Link]

Bernhardt, P. A. and C. L. Siefring, 2006: New satellite based systems for ionospheric tomography and scintillation region imaging. Radio Sci., 41, RS5S23, doi: 10.1029/2005 RS003360. [Link]

Bernhardt, P. A., R. P. McCoy, K. F. Dymond, J. M. Picone, R. R. Meier, F. Kamalabadi, D. M. Cotton, S. Chakrabarti, T. A. Cook, J. S. Vickers, A. W. Stephan, L. Kersely, S. E. Pryse, I. K. Walker, C. N. Mitchell, P. R. Straus, H. Na, C. Biswas, G. S. Bust, G. R. Kronschnabl, and T. D. Raymund, 1998: Two-dimensional mapping of the plasma density in the upper atmosphere with computerized ionospheric tomography (CIT). Phys. Plasmas, 5, 2010-2021, doi: 10.1063/1.872872. [Link]

Bernhardt, P. A., C. A. Selcher, S. Basu, G. Bust, and S. C. Reising, 2000: Atmospheric studies with the tri-band beacon instrument on the COSMIC constellation. Terr. Atmos. Ocean. Sci., 11, 291-312.

Fehmers, G. C., 1994: A new algorithm for ionospheric tomography, in Proceedings of the International Beacon Satellite Symposium, 52-55.
Franke, S. J., K. C. Yeh, E. S. Andreeva, and V. E. Kunitsyn, 2003: A study of the equatorial anomaly ionosphere using tomographic images. Radio Sci., 38, 1011, doi: 10.1029/ 2002RS002657. [Link]

Fremouw, E. J., J. A. Secan, and B. M. Howe, 1992: Application of stochastic inverse theory to ionospheric tomography. Radio Sci., 27, 721-732, doi: 10.1029/92RS00515. [Link]

Gringauz, K. I., 1958: Rocket measurements of the electron concentration in the ionosphere by means of an ultra-short wave dispersion interferometer. Sov. Phys. Dokl., 3, 620623.

Hanson, W. B. and R. J. Moffett, 1966: Ionization transport effects in the equatorial $\mathrm{F}$ region. J. Geophys. Res., 71, 5559-5572.

Kelley, M. C., J. J. Makela, J. L. Chau, and M. J. Nicolls, 2003: Penetration of the solar wind electric field into the magnetosphere/ionosphere system. Geophys. Res. Lett., 30, 1158, doi: 10.1029/2002GL016321. [Link]

Kersley, L. and S. E. Pryse, 1994: Development of experimental ionospheric tomography. Int. J. Imaging Syst. Technol., 5, 141-147, doi: 10.1002/ima.1850050210. [Link]

Kronschnabl, G. R., G. S. Bust, J. A. Cook, and C. J. Vasicek, 1995: Mid-America computerized ionospheric tomography experiment (MACE '93). Radio Sci., 30, 105-108, doi: 10.1029/94RS01538. [Link]

Kunitsyn, V. E. and E. D. Tereschenko, 1992: Radio tomography of the ionosphere. IEEE Trans. Antennas Propag., 34, 22-32, doi: 10.1109/74.163807. [Link]

Kunitsyn, V. E., E. S. Andreeva, O. G. Razinkov, and E. D. Tereschenko, 1994: Phase and phase-difference ionospheric radio tomography. Int. J. Imaging Syst. Technol., 5, 128140, doi: 10.1002/ima.1850050209. [Link]

Lawrence Livermore Laboratory, 1977: Numerical Electromagnetic Code (NEC-1) Part III: NEC User's guide.

Moffet, R., 1979: The equatorial anomaly in the electron distribution of the terrestrial R-region. Fundam. Cosmic Phys., 4, 313-391.

$\mathrm{Na}, \mathrm{H}$. and H. Lee, 1991: Orthogonal decomposition technique for ionospheric tomography. Int. J. Imaging Syst. Technol., 3, 354-365, doi: 10.1002/ima.1850030407. [Link]

Pakula, W. A., P. F. Fougere, J. A. Kobuchar, H. J. Kuenzler, M. J. Buonsanto, J. M. Roth, J. C. Foster, and R. E. Sheehan, 1995: Tomographic reconstruction of the ionosphere over North America with comparisons to ground-based radar. Radio Sci., 30, 89-103, doi: 10.1029/94RS02225. [Link]

Pryse, S. E. and L. Kersley, 1992: A preliminary experimental test of ionospheric tomography. J. Atmos. Terr. Phys., 54, 1007-1012, doi: 10.1016/0021-9169(92)90067-U. [Link]

Raymund, T. D., Y. Bresler, D. N. Anderson, and R. E. Daniell, 1994: Model-assisted ionospheric tomography: A new algorithm. Radio Sci., 29, 1493-1512, doi: 10.1029/94RS 01537. [Link]

Tsai, L. C., C. H. Liu, W. H. Tsai, and C. T. Liu, 2002: Tomographic imaging of the ionosphere using the GPS/MET 
and NNSS data. J. Atmos. Sol.-Terr. Phys., 64, 2003-2011, doi: 10.1016/S1364-6826(02)00218-3. [Link]

Yeh, K. C., S. J. Franke, E. S. Andreeva, and V. E. Kunitsyn,
2001: An investigation of motions of the equatorial anomaly crest. Geophys. Res. Lett., 28, 4517-4520, doi: 10.1029/2001GL013897. [Link] 